\title{
Perceptions of mental illness among Muslim general practitioners in South Africa
}

\author{
Z Mohamed-Kaloo, MA (Clinical Psychology); S Laher, PhD \\ Department of Psychology, School of Human and Community Development, University of the Witwatersrand, Johannesburg, South Africa
}

Corresponding author: S Laher (sumaya.laher@wits.ac.za)

\begin{abstract}
Background. Mental health literacy on the part of medical practitioners is an important component of mental healthcare. General practitioners (GPs) are typically the first doctors consulted by a person who is ill. Exploration of their perceptions regarding mental illness, aetiological issues and treatment is important.

Objective. To investigate perceptions of mental illness in a sample of 10 South African Muslim GPs (five male, five female) in the Lenasia area (Johannesburg, South Africa).

Methods. Using a qualitative approach, semi-structured interviews were conducted with each GP. The questionnaire encompassed 37 questions relating to the context in which the GPs practised, perceptions of mental illness, understanding of religion and culture, and treatment of mental illness (including aspects of spiritual illness). Thematic content analysis was used to analyse the data.

Results. Six dominant themes were identified, namely GPs' understanding of mental illness and its causation; stigma, secrecy and somatisation; the beneficial effects of religion in mental illnesses; perceptions of spiritual illnesses; collaboration with traditional healers; and collaboration with psychiatrists and psychologists.

Conclusion. Greater awareness regarding the stigmatisation of mental illness is needed. Furthermore, it is important that healthcare professionals have an understanding of religious and cultural taxonomies of illness and the use of traditional healing as a mode of treatment. Participants identified a need for increased collaboration between healthcare professionals, including traditional healers.
\end{abstract}

S Afr Med J 2014;104(5):350-352. DOI:10.7196/SAMJ.7863

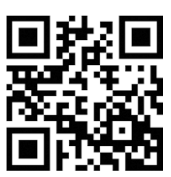

Local and international research consistently emphasises the importance of mental health literacy on the part of healthcare professionals and community members. Mental health literacy extends beyond the biopsychosocial sphere and calls for a greater awareness of religious and cultural values that can influence healthcare professionals and their patients. General practitioners (GPs) are often the first point of entry when people seek medical treatment, and their perceptions of mental illness, aetiological issues and treatment are important.

An examination of current research in the South African (SA) context indicates that research has focused on the prevalence of mental illness generally and across different groups. ${ }^{[1]}$ It has also focused on assessing the role of traditional healers and traditional healing in relation to more conventional care. ${ }^{[2]}$ Issues of mental health literacy ${ }^{[3]}$ and explanatory models of mental illness ${ }^{[4]}$ have been addressed. This research has mainly been quantitative. Qualitative studies have examined perceptions of mental illness in samples of traditional healers, volunteer counsellors, community members, psychologists and psychiatrists, but not among GPs. ${ }^{[5,6]}$ The GP, often the family physician and the community doctor, is typically the first professional from whom a person who is ill seeks treatment and healthcare advice.

According to $\mathrm{Ng},{ }^{[7]}$ culture can often influence mental illness in terms of perception, conception, experience of symptoms, classification, treatment, recognition, labelling and the course of mental illness. This is particularly the case in the SA context, where supernatural, religious, magical and moralistic approaches to mental illness exist. ${ }^{[5-7]}$ Spiritual punishment or sorcery is often identified as a cause of illness. ${ }^{[5,7]}$ It is therefore essential that GPs' perceptions of mental illness be explored, as they deal with patients from various cultures and religions and are well positioned to inform research on mental illness in SA.

\section{Objective}

We sought to investigate perceptions of mental illness among SA Muslim GPs, and to do this qualitatively to provide a richer description of their experiences.

\section{Methods \\ Participants}

A non-probability, convenience sample of ten Muslim general practitioners (five male and five female) in private practice in the Lenasia area of Johannesburg were interviewed. Participants were selected from Lenasia because it has the largest Muslim community in Gauteng Province. Members of the community provided the names of GPs who were most frequently consulted. GPs who were well established in the community were therefore selected for possible interview, and the first few who were interviewed provided referrals to other GPs practising in the area, permitting 'snowballing sampling' to obtain additional participants. GPs were contacted telephonically, and the first five females and five males who agreed to participate in the study were interviewed. A balanced sample in terms of patient gender was obtained because female patients generally visit female practitioners and male patients visit male practitioners. The sample consisted of both recently graduated and experienced doctors, with time in practice ranging from 1.5 to 40 years (mean 19.6).

\section{Instruments}

Semi-structured interviews approximately 45 minutes in length were conducted with each participant. The interview schedule was developed by consulting the relevant literature as well as four interview schedules from previous studies, ${ }^{[6,8-10]}$ and consisted of 37 questions covering contextual issues, GPs' perceptions of mental illness, understanding of religion and culture, questions on the treatment of mental illness and questions on spiritual illness. 


\section{Procedure}

Ethics clearance was obtained from the Human Research Ethics Committee (HREC Non-Medical) of the University of the Witwatersrand, Johannesburg (protocol no. MCLIN/11/004 IH). Participants were contacted telephonically and were informed regarding the nature and purpose of the study. The interviews were conducted with those participants who consented to be interviewed, at a place and time convenient to the participant. All interviews were audio-recorded and then transcribed.

\section{Data analysis}

Thematic content analysis as described by Braun and Clarke ${ }^{[1]}$ was used to analyse the data. Thematic content analysis aims to determine themes or patterns in data and involves reading and transcribing of the data, generation of initial codes, assembling codes into potential themes, generating a thematic map, and defining and naming themes. Transcripts were analysed using the ATLAS TI program.

\section{Results and discussion}

Six dominant themes were identified in the analysis. The first theme centred around GPs' understanding of mental illness and its causation. The second theme considered issues of stigma, secrecy and somatisation. The third theme focused on the beneficial effects of religion in the treatment of mental illnesses, while the fourth centred around GPs' perceptions of spiritual illnesses. Collaboration with traditional healers and collaboration with psychiatrists and psychologists were the final two themes identified. These themes are presented and discussed.

\section{GPs' understanding of mental illness and its cause}

Sixty per cent $(n=6)$ of the participants understood mental illness as any condition that affects one's daily functioning and mental wellbeing. Thirty per cent $(n=3)$ provided an intellectual understanding of mental illness based on their medical exposure and training and therefore defined it in terms of specific disorders (i.e. depression, anxiety, bipolar disorder, schizophrenia, etc.). Generally, participants' understanding of mental illness was based on the Diagnostic and Statistical Manual of Mental Disorders (DSM) or International Classification of Diseases (ICD) classification. This understanding of mental illness corresponded with participants' belief that in addition to organic causes, stress and lifestyle had a major influence on the development of mental conditions, most notably anxiety, depression and stress disorders. Participants elaborated on the above, stating that family issues have a serious impact on patients' well-being in the Lenasia community (reflecting financial pressures on married couples, both of whom often need to work, limited family time, marital conflicts, or changes in marital status such as divorce or death of a spouse). This concurs with Chiu et al. $\mathbf{s}^{[8]}$ finding that Canadian Asian women reported becoming mentally ill due to their extremely stressful lifestyles when they were expected to work, take care of children and do household chores. Marital conflict and relationships with in-laws were exacerbating factors. The typical busy Western lifestyle in Canada resulted in lack of family time and absence of social support from neighbours and friends, in contrast to lifestyles in India, where the community often gave support. ${ }^{[8]}$ These results concur with studies in the SA context, where psychosocial factors, notably stress, have been identified as a major cause of mental disorders. ${ }^{[3,9]}$

\section{Stigma, secrecy and somatisation}

Most participants acknowledged that mental illness is often stigmatised in the Lenasia community. All mentioned that their patients tended to keep mental illness a secret from their families as well as from the community at large. Patients were even reluctant to disclose their mental status to their GP, and concealing mental illness was common in the Indian Muslim community. Participants also mentioned the embarrassment faced by families when a member has a mental condition. Mental illness is therefore often disguised as a medical illness (e.g. diabetes or hypertension), which is more acceptable because it carries less stigma. Participants also stated that patients often present with pain-related complaints such as headaches, backaches or abdominal pain; however, these symptoms are not due to a medical condition but caused by underlying emotional distress.

According to Greenwood et al., ${ }^{[10]}$ Asian patients may not be referred for psychiatric care because they are prone to somatise psychological problems and consequently mental illnesses are not identified and diagnosed. These findings concur with reports by Chiu et al ${ }^{[8]}$ and Jacob et al. ${ }^{[12]}$ that the tendency to hide illness often resulted in GPs failing to detect mental illness, and many patients either being undiagnosed or misdiagnosed. Jacob et al. ${ }^{[12]}$ also found that doctors often believe that mental illness is managed within the family in Asian communities. A similar tendency was revealed in the current study, with a few of our participants mentioning that in the Indian Muslim population, people tend to rely on their extended families for support.

Together with the above factors, fear of stigmatisation may prevent people from seeking help. ${ }^{[3]}$ Participants in the current study supported this, and indicated that members of the Lenasia community also lack information about mental illness, which in turn contributes to stigmatisation. This concurs with the finding of Hugo et al.$^{[3]}$ that misinformation about mental illness led to stigmatisation of mental illness in the Cape Town community. Given the high prevalence of stigmatisation and lack of knowledge about mental illness, the participants in our study felt that campaigns to increase awareness were needed in Lenasia. Similarly, Mavundla et al. ${ }^{[13]}$ and Sorsdahl and Stein ${ }^{[9]}$ have recommended that educational programmes are needed to inform communities about the causes of mental illness, coping methods and the importance of counselling and medication, as well as cultural conceptualisations of mental illness.

\section{Beneficial effects of religion on mental illness}

Some participants $(n=5)$ in the current study reported that the Muslim community's religious beliefs, the teachings that Islam prescribes and their faith in the will of God makes it easier for Muslims to deal with issues. They felt that such faith reduces the prevalence of mental illnesses such as depression and substance abuse. Islam also assists by prohibiting (ab)use of alcohol and substances. Participants also acknowledged the potential benefit of religious practices such as dua (supplication), zikr (meditation) and salaah (ritual prayer, the second pillar of Islam). According to Carter and Rashidi, ${ }^{[14]}$ meditation and prayers have been used to promote and enhance emotional experiences as well as to establish a connection with God.

A few participants $(n=2)$ were of the opinion that patients may find it easier to relate to their GP and disclose their problems if disclosing to a GP from the same culture as themselves. Cinnirella and Loewenthal ${ }^{[15]}$ found that patients preferred GPs who were of the same religion, because they could recommend prayers that would assist in treating the ailment.

\section{GPs' beliefs regarding spiritual illness: Understanding aetiology and treatment}

The majority of the participants in this study $(n=8)$ acknowledged that spiritual illness exists, and patients often discussed experiences 
of such illnesses with their GPs. Spiritual illness was understood by the participants to mean symptoms that were beyond medical classification and therefore supernatural, such as jadoo (witchcraft), nazr ('evil eye') and jinn (spirits). Four participants mentioned that spiritual illness is common in the black African population, and that spiritual illness and traditional healing form part of SA culture. Recent studies have found that members of the black SA community often attribute mental health problems to cultural causes, such as witchcraft or the ancestors, rather than genetic or biological causes. ${ }^{[2,5,13]}$ Previous studies, locally and internationally, have also acknowledged the existence of a belief in witchcraft, the 'evil eye' and spirit possession in the Indian and/or Muslim populations, and the consequent belief that mental illness is a cause of such spiritual afflictions. ${ }^{[5,6,14]}$

\section{Collaboration with traditional healers}

Participants reported that in addition to consulting medical professionals, patients regularly consulted traditional healers such as maulanas (Muslim clergy) and sangomas (African traditional healers). It has been documented that a significant proportion of people in SA who suffer from mental health problems consult traditional healers. ${ }^{[2,5]}$

The participants noted that collaboration with and referral to traditional healers are important. According to Sorsdahl et al., ${ }^{[2]}$ SA has limited psychiatric care facilities and it may therefore be beneficial to work with traditional healers who are knowledgeable about cultural norms. Williams et al ${ }^{[1]}$ reported that in SA there are four psychiatrists, eight psychiatric nurses, four psychologists and 20 social workers per 100000 of the population. According to Sohrsdahl et al., ${ }^{[2]}$ there are at least 200000 traditional healers (1 per 500 population). Participants in this study also indicated that alternative forms of healthcare can be more accessible to patients than Western modes of treatment, as they are often cheaper. This is supported by the findings of Sorsdahl et al. ${ }^{[2]}$

\section{Collaboration with psychologists and psychiatrists}

All the participants agreed that they would refer patients to a psychiatrist if the need arose. However, they emphasised that patients were often reluctant to see psychiatrists and psychologists, preferring to be treated by their GP. Furthermore, three participants very rarely referred patients to psychiatrists, preferring psychologists because patients 'just needed a space to talk'. This is in agreement with previous findings in the SA context, ${ }^{[2,9]}$ which have indicated that people prefer to receive counselling rather than medication, particularly for mental illnesses such as depression.

The participants also mentioned that in the Indian Muslim community there may be stigma associated with seeing a psychologist or psychiatrist, as doing so is equated with madness, added to which are cultural expectations that emotional difficulties should be handled without professional help. A further factor preventing patients from seeking help is concern over confidentiality.

\section{Study limitations}

The findings of this study are based on a convenience sample of Muslim GPs from a specific area, Lenasia. The transferability of the results is therefore limited. The sample size was also small. However, the study allowed for an initial in-depth exploration of perceptions of mental illness. Similar studies with bigger and more representative samples will be useful in further research.

\section{Conclusion}

The participants stressed the importance of community awareness regarding mental illness in the form of educational interventions aimed at enhancing knowledge on the part of both GPs and the general public, with the intention of diminishing stigma. It is clear that cultural and religious taxonomies of illness need further exploration, especially as they pertain to spiritual illnesses. There is a need for greater collaboration between healthcare professionals, including traditional healers. Opportunities for the above should be made available through various continuing professional development activities or via conferences. The Health Professions Council of South Africa could play an important role in initiating such activities. There is also a need for further qualitative research to provide a richer understanding of the role of culture and religion in the understanding, aetiology and treatment of mental disorders, which can then inform training and intervention in the SA context.

\section{References}

1. Williams DR, Herman A, Stein DJ, et al. Twelve-month mental disorders in South Africa: Prevalence, service use and demographic correlates in the population-based South African Stress and Health Study. Psychol Med 2008;38(2):211-220. [http://dx.doi.org/10.1017/S0033291707001420]

2. Sorsdahl K, Stein DJ, Grimsrud A, et al. Traditional healers in the treatment of common mental disorders in South Africa. J Nerv Ment Dis 2009;197(6):434-441. [http://dx.doi.org/10.1097/ NMD.0b013e3181a61dbc]

3. Hugo CJ, Boshoff DEL, Traut A, Zungu-Dirwayi N, Stein DJ. Community attitudes toward and Hugo CJ, Boshoff DEL, Traut A, Zungu-Dirwayi N, Stein DJ. Community attitudes toward and
knowledge of mental illness in South Africa. Soc Psychiatry Psychiatr Epidemiol 2003;38(12):715-719. [http://dx.doi.org/10.1007/s00127-003-0695-3]

[http://dx.doi.org/ 10.1007/s00127-003-0695-3]
Petersen I, Lund C. Mental health service delivery in South Africa from 2000 to 2010: One step 4. Petersen I, Lund C. Mental health service delivery in So
forward, one step back. S Afr Med J 2011;101(10):751-757.

5. Ally Y, Laher S. South African Muslim faith healers perceptions of mental illness: Understanding aetiology and treatment. J Relig Health 2008;47(1):45-56. [http://dx.doi.org/10.1007/s10943-007-9133-2] 6ulbulia T, Laher S. Perceptions of mental illness among South African Muslim psychiatrists. South African Journal of Psychiatry 2013;19(2):52-54.

7. Ng C. The stigma of mental illness in Asian cultures. Aust N Z J Psychiatry 1997;31(3):382-392. [http:// dx.doi.org/10.3109/00048679709073848]

8. Chiu L, Ganesan S, Clark N, Morrow M. Spirituality and treatment choices by South and East Asian women with serious mental illness. Transcult Psychiatry 2005;42(4):630-656. [http://dx.doi. org $/ 10.1177 / 1363461505058920$

9. Sorsdahl K, Stein DJ. Knowledge of and stigma associated with mental disorders in a South African community sample. J Nerv Ment Dis 2010;198(10):742-747. [http://dx.doi.org/10.1097/ Arican com

10. Greenwood N, Hussain F, Burns T, Raphael F. Asian in-patient and carer views of mental health care: Asian Greenwood N, Hussain F, Burns T, Raphael F. Asian in-patient and carer views of mental health care: Asian
views of mental health care. J Ment Health 2000;9(4):397-408. [http://dx.doi.org/10.1080/jmh.9.4.397.408] 1. Braun V, Clarke V. Using thematic analysis in psychology. Qual Res Psychol 2006;3(2):77-101. [http:// dx.doi.org/10.1191/1478088706qp063oa]

12. Jacob KS, Bhugra D, Lloyd KR, Hannah AH. Common mental disorders, explanatory models and consultation behaviour among Indian women living in the UK. J R Soc Med 1998;91(2):66-71.

13. Mavundla TR, Toth F, Mphelane ML. Caregiver experience in mental illness: A perspective from a rural community in South Africa. Int J Ment Health Nurs 2009;18(5):357-367. [http://dx.doi. org/10.1111/j.1447-0349.2009.00624.x

14. Carter DJ, Rashidi A. Theoretical model of psychotherapy: Eastern Asian-Islamic women with mental illness. Health Care Women Int 2003;24(5):399-413. [http://dx.doi.org/10.1080/07399330390212180]

15. Cinnirella M, Loewenthal K. Religious and ethnic group influences on beliefs about menta illness: A qualitative interview study. Br J Med Psychol 1999;72(4):505-524. [http://dx.doi. org/10.1348/000711299160202]

Accepted 20 January 2014. 